\title{
Non Invasive Prenatal Testing- Indian Scenario
}

\author{
Mala Srivasatava*1 $^{* 1}$ and Ankita Srivastava ${ }^{2}$ \\ ${ }^{1}$ Senior Consultant and Robotic Surgeon, Institute of Obstetrics and Gynaecology, India \\ ${ }^{2}$ Senior Resident, Department of Obstetrics and Gynaecology, India
}

Received: February 21, 2018; Published: February 28, 2018

*Corresponding author: Mala Srivasatava, Senior Consultant and Robotic Surgeon, Institute of Obstetrics and Gynaecology, Sir Ganga Ram Hospital, New Delhi, India, Email: malasrivastava2001@yahoo.co.in

\begin{abstract}
Abbrevations: ID: Intellectual Disability; ACOG: American College of Obstetrics and Gynecologists; NT: Fetal Nuchal Translucency; PAPP-A: Pregnancy Associated Plasma Protein-A; NIPT: Non Invasive Prenatal Testing; b-HCG: Beta Human Chorionic Gonadotropin; CVS: Chorionic Villus Sampling; Positive Predictive Value
\end{abstract}

\section{Introduction}

Intellectual disability (ID) affects about $3 \%$ of the general population. Chromosomal and genetic disorders account for 30$40 \%$ of moderate to severe IDs, of which, Down syndrome is the commonest. Attempts to detect and prevent the birth of such affected children were initiated in the ' 80 s but were initially limited to women in the advance maternal age. Current recommendations by the American College of Obstetrics and Gynecologists (ACOG) Committee, are that everywoman, regardless of maternal age, should be offered prenatal assessment for aneuploidy either by screening or invasive prenatal diagnosis [1]. The incidence of Down syndrome is $1: 800$, so approximately 32,000 babies with Down syndrome are born every year in India (the birth rate of India is 25.6 million births annually). Though screening facilities are available in most of the cities, expertise in invasive testing is limited, thus affecting the overall utility of screening. Current screening tests have a detection rate of $95 \%$ with a false positive rate of $5 \%$, when maternal age, fetal nuchal translucency (NT), nasal bone, and maternal serum markers like beta human chorionic gonadotropin (b-hCG) and pregnancy associated plasma protein A (PAPP-A) are combined together [2].

Women in the high-risk group require confirmatory diagnostic testing either via chorionic villus sampling (CVS) or amniocentesis. These procedures are associated with risk of abortion of $0.2-1$ $\%$, which may result in the potential loss of a healthy fetus. This initiated efforts to develop a noninvasive method with better sensitivity and specificity, and it is now available for clinical use. This test is termed the noninvasive prenatal testing (NIPT). This technology involves testing the cells free fetal DNA which is present in the maternal blood [3]. Noninvasive prenatal screening for aneuploidies based on cell-free fetal DNA in maternal blood (NIPS) was introduced as a service in the USA in 2011. This test rapidly spread like wild fire, irreversibly changing prenatal screening, and markedly reducing the rate of invasive procedures such as chorionic villus sampling and amniocentesis. A large number of studies were done in low-risk women, as well as going beyond aneuploidies to microdeletions and whole genome copy number variations; ACMG issued an update on this topic [4].

The updated statement points out that NIPT is a screening test, and not a diagnostic test, so it is more appropriate to call it NIPS i.e. non invasive prenatal screening. As it is a screening test, there remains a residual risk of disease, even if the test is reported normal; which means that a normal result on NIPS does not completely exclude the presence of aneuploidy. In one large study the residual risk for aneuploidies, or false negative rate was 9/11,103 i.e. $0.008 \%$. NIPS is a high efficiency screening test, based on the metaanalysis of 37 relevant studies, that showed a sensitivity and false positive rate of 99.2 and $0.09 \%$ for trisomy $21,96.3$ and $0.18 \%$ for trisomy $18,91.0$ and $0.13 \%$ for trisomy $13,90.3$ and $0.23 \%$ for monosomy X, 93 and $0.14 \%$ for the sex chromosomal abnormalities. NIPS being a screening test so in the event of a positive NIPS test, the diagnosis need to be confirmed by an invasive procedure such as chorionic villus sampling or amniocentesis [5].

In the event of a high risk for Down syndrome, aneuploidy of other chromosomes, or other cytogenetic abnormalities on NIPS, the pregnancy should never be terminated, but a diagnostic test should be performed instead. The patient should be clearly told what NIPS does not provide. NIPS cannot be used to detect all chromosomal abnormalities, missing out on abnormalities of chromosomes other than 21, 18, 13 and sex chromosomes, as well as structural abnormalities and translocations. It does not 
diagnose congenital malformations like spina bifida or congenital heart disease, it cannot predict abnormal pregnancy outcomes like growth restriction or preeclampsia, and it does not, at present, test for single gene disorders. NIPS also cannot replace imaging by ultrasound studies. Numerous validation studies have been carried out, and they have established that NIPS has very high detection rate and specificity, reaching nearly 99\% for Down syndrome caused by trisomy 21, translocations, and trisomy 21 mosaicism; 80-100\% for Edwards syndrome caused by trisomy 18 and trisomy 18 mosaicism, as well as for Patau syndrome caused by trisomy 13 , translocations and trisomy 13 mosaicism.

The main objective of the pre-test genetic counseling is to provide the patient information on different methods of prenatal diagnosis, and what each test is capable of diagnosing. The benefits and disadvantages of various invasive procedures as well as noninvasive tests should be explained, so that the patients can take an informed decision as to which test is most appropriate for their situation. ACMG also recommends that women at high risk of aneuploidy, either on biochemical tests or on ultrasound findings, should be given the option of diagnostic or invasive testing for detection of chromosomal abnormality, in place of going through the process of NIPS. If the combined test risk is more than 1 in 50 , invasive testing would be appropriate, rather than NIPS. In women over 38 years NIPT is recommended rather than biochemical screening, unless there are some contraindications for NIPT, such as advanced gestation beyond the time for termination under Indian law (20 weeks), or patient is from a far-off place making it difficult for the patient to return for a re-test. In such circumstances diagnostic testing through an invasive procedure is recommended.

Additional benefits of invasive testing are that abnormalities of all chromosomes are revealed, including translocations and deletions. Microarray studies can also be done on fetal cells when indicated e.g., in the presence of an abnormality on the ultrasound studies, as these reveal a background rate of clinically significant copy number variations (CNVs) to be $1-2 \%$. The risk of miscarriage and other complications of invasive procedures are much lower (for amniocentesis 1 in 909, and for chronic villus sampling 1 in 454 [6].

ACMG states that the companies offering this test should make NIPS accessible to all pregnant women. Women often report for genetic tests at a later stage in gestation (say more than 19 weeks). In such an event NIPS is not a good option, considering that the report will not be available for 8-10 days, and the legal limit for termination of pregnancy in India is 20 weeks. Secondly, there is problem in offering NIPS to patients from the peripheral towns where there are no facilities for collection of blood for NIPS testing, or those who come from a great distance which makes it difficult for them to return for a repeat test, in the event of a no call on the test report.

The NIPT is ideally offered during the first trimester, as there is sufficient time for the report or a repeat sample if it becomes necessary. Initially NIPS was offered to only high-risk women. Subsequently experience has been gained in performing NIPS in low-risk women. The sensitivity of NIPS is greater than the traditionally used screening options for detecting aneuploidies in low-risk women. Its positive predictive value (PPV) is also higher than the traditional biochemical screening and ultrasound markers [7]. ACMG recommends that all pregnant women be informed that NIPS is the most sensitive screening test for the traditional aneuploidies (trisomy 21, 18, and 13). NIPS becomes affordable, then it can be offered to women at low risk of aneuploidies, as it has a better sensitivity, specificity and positive predictable value. Keeping the high cost of NIPS tests in mind, the most appropriate and affordable protocol in India would be to carry out combined test (dual marker test-PAPP-A and free bhCG plus NT), and in those with high risk be offered NIPS, except that in those with very high risk (say more than 1 in 50 ) invasive testing may be offered.

When an increased risk of aneuploidy is reported after NIPS, it is essential that the patient be counselled that diagnostic testing is essential. In no case should a woman with a high-risk NIPT be allowed to proceed to termination of pregnancy. If the invasive testing confirms the presence of a chromosomal disease balanced information should be provided. ACMG recommends that laboratory reports should clearly state the detection rate, specificity, positive and negative predictive value of their tests. Some laboratories provide screening for other chromosomal aneuploidies such as for chromosome 16 or 22. ACMG does not recommend screening for aneuploidies other than trisomy 21,18 , and 13 , because of lack of sufficient validation of testing for these extra autosomes, as well as their clinical value. One important issue with NIPT is the frequency of "no call" for the report. This is often due to a low fetal fraction or the test having been performed in a very obese woman, or very early in pregnancy (less than 9 weeks). It is generally agreed that for an accurate and reliable report the minimum fetal fraction should be $4 \%$, and below this amount of fetal fraction the report may be unreliable [8].

This might be appropriate if the NIPT has been performed early in pregnancy (less than 12 weeks), as there is adequate time for getting the results. However, from 12 to 20 weeks the fetal fraction increases less than $0.1 \%$ per week, which challenges the idea that repeating sample collection is useful in cases of no call due to low fetal fraction. Therefore, ACMG recommends that such women be offered diagnostic testing. As fetal fraction is very critical to obtain an accurate result ACMG recommends that all laboratories should state the fetal fraction in their NIPS reports. One cannot confidently rely on tests that do not state fetal fraction [9]. ACMG recommends that all laboratories should state the reason for a "nocall" in their reports. Two common reasons are a low fetal fraction as stated above or technical reasons. In India and other countries with a high rate of consanguinity there may be long stretches of homozygosity in the DNA and this may be the reason for a no call in tests performed on SNP analysis. However whenever stretches of homozygosity are reported the geneticist should look into the possibility of uniparental disomy which may lead to the unmasking of a recessive disorder [10]. 
Some countries offer NIPT screening only for the three aneuploidies, and consider that screening for sex chromosomes is not necessary, as syndromes such as XXY and XYY are compatible with a reasonably good life. In India couples prefer to know abnormalities of the sex chromosomes as they desire the baby should not face "any" problems after birth. The positive predictive value of NIPT for sex chromosomal abnormalities ranges from 38 to $67 \%$, and is lower than that for the autosomal aneuploidies [11]. Determination of sex is not permitted by law in India except when there is X-linked disease. In India the laboratories screen for sex chromosomes but do not report sex of the baby so as to comply with the Indian law. However they do report if there is an abnormality of the sex chromosomes. If the presence of a sex chromosomal disorder is confirmed the couple should be provided accurate information. A number of studies have been carried out using NIPS to detect microdeletions. Unfortunately, these tests have not been properly validated due to the lack of availability of sufficient number of patients having microdeletions. In one large study, consisting of about 21,000 women the PPV was only $19 \%$ (varying from 11 to $48 \%$ in different studies).

ACMG recommends that all pregnant women be informed about the availability of tests to detect microdeletions, and also be told about the low positive predictive values of these tests, as well as greater false positive and false negative results. The couple should be informed that even more detailed information would be available by study of fetal DNA, obtained through an invasive procedure, using microarrays. If the NIPT detects a microdeletion this must be confirmed by an invasive test [12]. Screening for CNV over the whole genome is not recommended by ACMG. If this level of information is desired, then diagnostic testing (e.g., chorionic villous sampling or amniocentesis) followed by a microarray study is recommended. With regard to pregnancies with multiple gestations and/or donor oocytes vendors who utilize the counting method after massively parallel sequencing, are successful in providing a report. The ACMG feels that more data on twins is required before use of NIPT can be recommended with confidence. Occasionally a bizarre pattern of DNA reads is obtained. Such cases have arisen from the presence of a malignancy in the pregnant woman, the aberrant DNA sequences being derived from the malignant cells. Inaccuracies in the NIPT report may be introduced if the pregnant woman is the recipient of a bone marrow transplant or has received blood transfusion within the prior 4 weeks of the test [13].

\section{Conclusion}

Noninvasive prenatal test qualifies as an 'advanced' screening test. Recently, false negative cases of NIPT have been published which emphasize that even though the sensitivity and specificity of NIPS is high, it is still a screening test and cannot replace the invasive diagnostic tests. The utility of NIPT is very high if done for correct indications. The acceptance of this test will be greater if the test could be made more economical. Applied selectively, it provides tremendous benefits even in developing countries.

\section{References}

1. Gustavson KH, Hagberg B, Hagberg G, Sars K (1977) Severe mental retardation in a Swedish county. II. Etioiogic and pathogenetic aspects of children born 1959-1970. Neuropadiatrle 8: 293-304.

2. Cuckle H, Benn P (2013) Multi-analyte maternal serum screening for chromosomal defects. In: Milunsky A, Milunsky JM (Eds.). Genetic disorders and the fetus: diagnosis, prevention and treatment. $6^{\text {th }}$ (Edn.). Chichester: (2010) Wiley-Blackwell, pp. 771-818.

3. Chitty LS, Bianchi DW (2013) Non-invasive prenatal testing: the paradigm is shifting rapidly. Prenat Diagn 33(6): 511-513.

4. Khalifeh A, Weiner S, Berghella V, Donnenfeld A (2016) Trends in invasive prenatal diagnosis: effect of sequential screening and noninvasive prenatal testing. Fetal Diagn Ther 39: 292-296.

5. Gil MM, Quezada MS, Revello R, Akolekar R, Nicolaides KH (2015) Analysis of cell-free DNA in maternal blood in screening for fetal aneuploidies: updated meta-analysis. Ultrasound Obstet Gynecol 45(3): 249-266.

6. Wapner RJ, Martin CL, Levy B, Blake C Ballif, Christine M Eng, et al. (2012) Chromosomal microarray versus karyotyping for prenatal diagnosis. $\mathrm{N}$ Engl J Med 367: 2175-2184.

7. Zhang H, Gao Y, Jiang F, Fu M, Yuan Y, et al. (2015) Non-invasive prenatal testing for trisomies 21,18 and 13: clinical experience from 146,958 pregnancies. Ultrasound Obstet Gynecol 45: 530-538.

8. Kinnings SL, Geis JA, Almasri E, Wang H, Guan X, et al. (2015) Factors affecting levels of circulating cell-free fetal DNA in maternal plasma and their implications for noninvasive prenatal testing. Prenat Diagn 35(8): 816-822.

9. Wang E, Batey A, Struble C, Musci T, Song K, et al. (2013) Gestational age and maternal weight effects on fetal cell-free DNA in maternal plasma. Prenat Diagn 33(7): 662-666.

10. Rehder CW, David KL, Hirsch B, Toriello HV, Wilson CM, et al. (2013) American College of Medical Genetics and Genomics: standards and guidelines for documenting suspected consanguinity as an incidental finding of genomic testing. Genet Med 15: 150-152.

11. Porreco RP, Garite TJ, Maurel K, Marusiak B, Ehrich M, et al. (2014) Noninvasive prenatal screening for fetal trisomies $21,18,13$ and the common sex chromosome aneuploidies from maternal blood using massively parallel genomic sequencing of DNA. Am J Obstet Gynecol 211(4): 365.e1-365.e12.

12. Wapner RJ, Babiarz JE, Levy B, Stosic M, Zimmermann B, et al. (2015) Expanding the scope of noninvasive prenatal testing: detection of fetal microdeletion syndromes. Am J Obstet Gynecol 212(3): 332.

13. Bianchi DW, Chudova D, Sehnert AJ, Bhatt S, Murray K, et al. (2015) Noninvasive prenatal testing and incidental detection of occult maternal malignancies. JAMA 314(2): 162-169. 


\section{(c) () This work is licensed under Creative}

Submission Link: http://biomedres.us/submit-manuscript.php

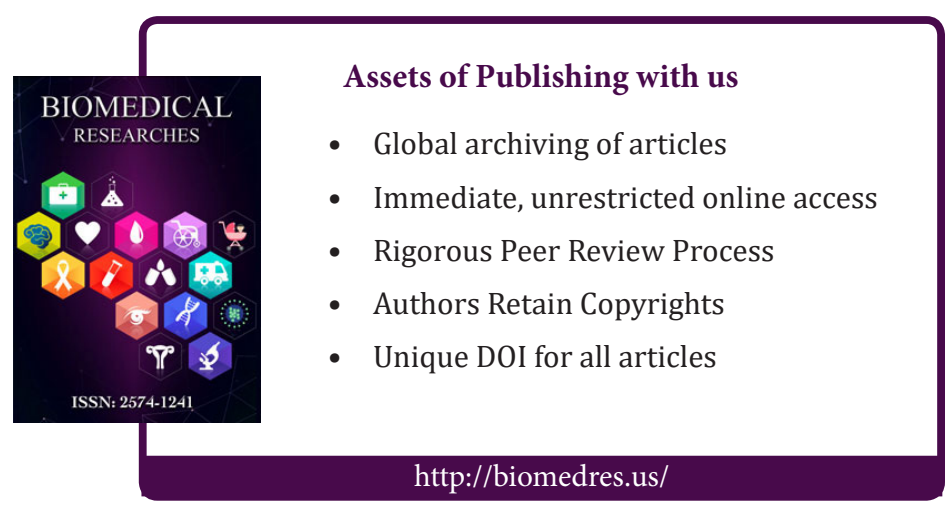

